\title{
Summen in der City und im Internet of Things
}

\section{Transformationspotenziale aus kulturwissenschaftlicher Perspektive}

Marie-Helene Wichmann, Institut für Kulturwissenschaft und Ethnologie (IfEK), Universität Bremen, Universitätsallee 1, 28359 Bremen

(m.wichmann@uni-bremen.de) (D) https://orcid.org/0000-0001-9765-7241

Mit kulturwissenschaftlich-ethnographischen Methoden und Daten untersucht dieser Beitrag die Transformationspotenziale des urbanen Imkerns für klimaneutrale und intelligente Städte, z. B. hinsichtlich der emissionsarmen urbanen Nahrungsmittelerzeugung oder der Bestäubungsleistung zugunsten urbaner Artenvielfalt. Im Ergebnis zeigt sich im gegenwärtigen Trend zur Honigbienenhaltung im Stadtraum eine Verknüpfung von Sozialinnovationen (gemeinschaftliches Imkern) und technologischen Innovationen (agrarinformatische Techniken und Anwendungen des Internet of Things, IOT). Beide Innovationen stehen im Spannungsfeld zwischen Naturschutzmotivationen auf der einen und agroindustriellen Ausrichtungen der digitalen Technologien für Imkernde auf der anderen Seite. Im Ergebnis zeigt sich, dass das Transformationspotenzial urbanen Imkerns insbesondere in der Vernetzung imkerlicher Daten sowie in der Einbindung naturschutzbezogener Daten durch Informations- und Kommunikationstechnologien liegt.

\section{Buzzing in the City and the Internet of Things \\ Transformational potentials of urban apiculture from \\ a social anthropological perspective}

Using ethnographic methods and data, this paper explores the transformational potential of urban beekeeping for climate-neutral and intelligent cities, e. g., in terms of low-emission urban food production or pollination services for urban biodiversity. As a result, the current trend of beekeeping in urban areas shows a combination of social innovations (community beekeeping) and technological innovations (agro-informatics techniques and Internet of Things applications). Both innovations are in tension between ecological motivations on the one hand and agro-industrial orientations of digital technologies for beekeepers on the other hand. The results show that the transformation potential of urban beekeeping lies in particular in the networking of beekeeping data and in the integration of nature conservation-related data through information and communication technologies.

This is an article distributed under the terms of the Creative Commons Attribution License CCBY 4.0 (https://creativecommons.org/licenses/by/4.0/)

https://doi.org/10.14512/tatup.30.1.50

Submitted: 20.09.2020. Peer reviewed. Accepted: 09. 02.2021
Keywords: urban agriculture, urban beekeeping, smart beehives, beekeeping data, ethnographic research

\section{Einführung}

Laut Deutschem Imkerbund leisten Bienen einen Beitrag zum Klimaschutz, indem sie zum einen durch Bestäubung ,die Bildung pflanzlicher Biomasse“ fördern, zum anderen die Kohlendioxid-Bilanz verbessern, indem sie durch Bestäubung Ertragssteigerungen bewirken, weiter, weil die Produkte Honig sowie Wachs klimaneutral erzeugt werden und zudem ,,jedes Bienenvolk [...] jährlich bis zu $15 \mathrm{~kg}$ Biomasse durch Bienenorganismen [bindet]“ (DIB 2020a, S. 7). Da mit Blick auf die fortschreitende Urbanisierung die städtische Produktion und Konsumption von Nahrungsmitteln und die Zukunftsvision einer nachhaltigen, an natürlichen Stoffkreisläufen orientierten Wirtschaft eine Rolle spielen, sind nicht nur Bienenprodukte, sondern auch die Ökosystemleistung von Bienen wichtige Teile der urbanen Agrikultur.

$\mathrm{Zu}$ den Bienenprodukten, die aus diesem Superorganismus (bestehend aus den einzeln nicht überlebensfähigen Bienenwesen samt Wabenwerk und eingelagerter Nahrung) entnommen werden können, zählen von den Bienen produzierte Stoffe wie Wachs und Gelee Royale, aber mit Honig, Propolis, Blütenpollen und Perga auch Produkte, die auf von Bienen gesammelten Inhaltsstoffen basieren. 20 bis 30 Kilo Honig, der von den Honigbienen aus Pflanzennektar unter Beimengung von Speichel eingedickt wird, können jährlich pro Bienenstock entnommen werden. Über die Bienenprodukte hinaus ist jedoch die Ökosystemleistung der Bestäubung von Nutz- und Wildpflanzen der urbanen Agrikultur kaum hoch genug einzuschätzen. Sowohl der Honigertrag als auch die Ökosystemleistung der Bestäubung sind von lokalen Bedingungen wie Wetter, Flora und Fauna abhängig. Die künftigen Auswirkungen des Klimawandels auf die Vegetation und damit auf das Nektar- und Pollenangebot werden auch die Honigbienen in ungewissem Ausmaß beeinflussen. Bereits feststellbar sind verbesserte Bedingungen 
für Bienenkrankheiten und -schädlinge als Folgen des Klimawandels (DIB 2020a, S. 8). Da Honigbienen in urban geprägten Räumen produktiver sind als in gemischten oder landwirtschaftlich genutzten Gebieten (Lecocq et al. 2015) und der Honig aus der Stadt auch weniger mit Schadstoffen belastet ist (von der Ohe et al. 2017), bieten sich Städte in Zeiten der multifaktoriellen Bedrohungslage von Insekten für die Bienenhaltung an. Kathryn Peters (2012, S. 642) argumentiert daher, dass mit den urbanen Gartenbauflächen auch die Honigbienenhaltung zunehmen sollte.

Dieser Beitrag fragt unter Berücksichtigung von Problemlagen des urbanen Imkerns nach dessen Transformationspoten- ten, hatten sich Imkerpraktiken kaum verändert, bis mit Ausbreitung der Varroamilbe in Deutschland seit den 1980er-Jahren Behandlungen zur Reduktion der Milbenzahl hinzukamen. Wie das Sammeln und Bestäuben hängen auch imkerliche Eingriffe in den Bienenstock, besonders die Milbenbehandlungen, von Witterungsfaktoren ab und sind damit vom Klimawandel betroffen. $\mathrm{Zu}$ unterschiedlichen imkerlichen Praktiken führen neben den verschiedenen Beuteformen auch die persönlichen Weltanschauungen, z. B. ökologische Einstellungen der Imker*innen. Wie meine Feldnotizen zeigen, sind imkerliche Praktiken somit höchst individuell und werden von Imkernden auch als solche kommuniziert.

\section{Das Sammelgebiet von Honigbienen umfasst bis zu 50 Quadratkilometer um die Beute.}

zial für klimaneutrale Städte. Untersuchungsgegenstand ist dabei insbesondere die Verknüpfung der Sozialinnovation des gemeinschaftlichen Bewirtschaftens von Honigbienen mit technologischen Innovationen, wie z. B. agrarinformatischen Anwendungen des Internet of Things (IoT). Die Untersuchung basiert auf 25 Monaten Feldforschung in Bremen. Während dieser Zeit wurden vor allem Einsteiger*innen ins gemeinschaftliche Imkern begleitet. In diesen Imkergruppen wurde die Arbeit mit Sensoren in und an Bienenbehausungen (Beuten) zur Datenerhebung und zur Unterstützung imkerlicher Entscheidungen erprobt. Die Datenbasis für die im folgenden präsentierten Ergebnisse bilden teilnehmende Beobachtungen, Experteninterviews und Gruppen-Diskussionen zusammen mit Inhalts-, Diskurs- und Nutzungsanalysen.

\section{Kulturwissenschaftliche Perspektiven auf die Stadtimkerei}

Überlieferungen und Grabungsfunde legen nahe, dass es Bienenhaltung in der Stadt bereits in der Antike gab (Epstein 2019, S. 65). Spezielle Beuten für Stadthäuser werden in der deutschen Imker-Literatur des 19. Jahrhunderts beschrieben (Dzierzon 1855). Dort finden sich auch bereits erste Ansätze für die gemeinschaftliche Bewirtschaftung von Honigbienen für die damals neue Zielgruppe der Frauen. Heute liegt der Frauenanteil im Deutschen Imkerbund bei 20\% (DIB 2020b). Die Imkerei als Agrarsektor ist in Deutschland durch Nebenerwerbs- und Hobbyimkerei geprägt. Praktiken des Imkerns richten sich nach den biologischen Zuständen und Abläufen im Superorganismus der Honigbienen. Imkerliche Arbeiten als Eingriffe in den Bienenstock erfordern vor allem im Frühling und Sommer, also in den Hauptferienzeiten, erheblichen Zeiteinsatz.

Seit dem 19. Jahrhundert, als sich Magazinbeuten aus stapelbaren Kästen mit beweglichen Rähmchen oder Leisten etablier-
Der aktuelle, seit der Jahrtausendwende anhaltende Stadtimkerei-Boom hat eine Vielzahl sozial- und kulturwissenschaftlicher Untersuchungen hervorgebracht (Fenske 2019; Heyer 2018; Lorenz und Stark 2015; Sulzner 2016; Moore und Kosut 2014). Diese zeigen, wie Stadtimkerei eng verflochten ist mit Befürchtungen über das potenzielle Aussterben der Schlüsselspezies Biene (Fenske 2017), Naturschutzbestrebungen und den Urban-Gardening- sowie Urban-Farming-Bewegungen (Fenske 2019; Peters 2012), die ihrerseits ökologische, soziale und gesundheitspolitische Themen wie Teilhabe, Gemeinschaftserleben (Community), Handlungsfähigkeit (Empowerment), Ernährungssicherheit sowie städtische Flächennutzung und -aneignung verknüpfen (Zezza und Tasciotti 2010; Müller 2011; Bornemann und Weiland 2019). Honigbienen verbinden diese Dimensionen ökologisch, ökonomisch und auch symbolisch.

\section{Spezifika des Stadtimkerns}

Die Bedingungen fürs Imkern in der Stadt weisen einige Besonderheiten auf. Bienenbeuten können in der Stadt auch auf Balkon- und Dachflächen, also wenig genutzten und teilweise schwer zugänglichen Flächen, aufgestellt werden. Stehen Bienenstöcke im öffentlich zugänglichen Raum wie in einer Grünanlage, können sie durch bauliche Maßnahmen wie einen Zaun oder ein Bienenhaus geschützt werden. Diese Zugangsbarrieren dienen dem Schutz der Bienenstöcke vor Diebstahl und Vandalismus.

Das Sammelgebiet von Honigbienen umfasst bis zu 50 Quadratkilometer um die Beute. Dies entspricht in Städten durchaus dem Gebiet mehrerer Stadtteile. Aufgrund der kleinteilig strukturierten, von verschiedenen Besitz- und Nutzungsverhältnissen geprägten Stadtlandschaft sind die Trachtverhältnisse, die Bienen vorfinden, für Imkernde kaum bis gar nicht kon- 
trollierbar. Welche Trachtquellen die Bienen eines Stocks anfliegen, entscheidet der Bienenstock. In urbanen Lebensräumen sind die für Sortenhonige notwendigen großen Trachtmengen selten, die in der konventionellen Landwirtschaft mit Monokulturflächen anzutreffen sind. Durch tägliche Fluglochbeobachtung - eine traditionelle Imkerpraktik mit erheblichem Zeitaufwand - sind zwar aufgrund der Farbe des eingetragenen Pollens Rückschlüsse auf die Trachtquellen möglich. Meist gibt aber erst eine Laboranalyse der Pollenbestandteile im Honig Aufschluss über dessen tatsächliche Zusammensetzung, die oft für eine Benennung des Honigs herangezogen wird. Die räumliche Nähe von Honigbienenständen ist auch für den Seuchenschutz relevant, da es hier durch den Kontakt von Bienen aus verschiedenen Bienenstöcken leichter zur Übertragung von Krankheiten kommen kann.

Das Durchschnittsalter von Imkernden liegt bei 56 Jahren, dahingegen beruht der Stadtimkerei-Trend vor allem auf dem Engagement jüngerer, umweltbewusster Bevölkerungsgruppen (DIB 2020 a, S. 12 ff.). Auch wenn der Deutsche Imkerbund jüngst einen leichten Rückgang der Stadtimkerei festgestellt hat, waren Kurse in der Stadt Bremen, in denen die praktischen und theoretischen Grundlagen des Imkerns vermittelt werden, weit im Voraus ausgebucht. Teilnehmer*innen von Imkerkursen entscheiden sich am Ende des Kurses nicht immer für eigene Bienen. Dies liegt unter anderem daran, dass viele imkerliche Tätigkeiten von Witterung und Wetter abhängen, weshalb die Zeitpunkte hierfür selten langfristig terminierbar, aber trotzdem auf konkrete Zeitfenster beschränkt sind. Imkern ist durch diese

\section{Smarte Imkereitechnologie}

Mit Precision Beekeeping oder Precision Apiculture hat sich beflügelt durch die multidimensionale Gefährdungslage der Honigbienen - eine agrarinformatische Forschungsrichtung des IoT etabliert. Sie greift für die Auswertung der entstehenden Datenmengen auf Anwendungen der künstlichen Intelligenz (KI) zurück (Dineva und Atanasova 2017; Zabasta et al. 2019) und hat diverse, modular aus kostengünstigen Komponenten zusammengesetzte Systeme sowie Anwendungen für die Unterstützung Imkernder mittels Sensorik entwickelt. Apps zur Bienenstandverwaltung nutzen retrospektive Monitoringdaten und verfügen über Schnittstellen zu Datenbanken mit postleitzahlengenauen Wetterbedingungen sowie Trachtpflanzenverhältnissen, auf deren Basis Empfehlungen für die angegebenen Bienenstandorte ausgegeben werden. Einige Systeme machen den Gesundheitszustand von Bienenstöcken ohne den Einbau von Sensorkomponenten in den Bienenstock in Echtzeit überprüfbar (Zabasta et al. 2019), etwa mittels Wifi-Verbindungen (Zacepins et al. 2016).

Start-ups bieten bereits Sensorsysteme an, die in Deutschland auf eine Imkereiwirtschaft stoßen, in der viele Nebenerwerbsund Hobbyimkernde einer vergleichsweise kleinen Zahl von Berufsimkernden gegenüberstehen. Ziel der Nutzung von Sensoren ist es, Imkernde durch Informationen über den Zustand der Bienenstöcke entfernungsunabhängig und ertragsorientiert $\mathrm{zu}$ unterstützen. Dieses Vorgehen entspricht den Zielsetzungen des traditionellen Precision Livestock Farmings aus anderen Bereichen der Landwirtschaft, das neben Ertragssteigerung und Zeit-

\section{Apps zur Bienenstandverwaltung machen den Gesundheitszustand von Bienenstöcken ohne den Einbau von Sensorkomponenten in den Bienenstock in Echtzeit überprüfbar.}

eingeschränkte Planbarkeit und den diskontinuierlichen Zeitaufwand, der vor allem die Sommermonate betrifft, schlecht mit urbanen Lebensstilen der mittleren Lebensphasen zu vereinbaren, weil diese oft neben festen Arbeitszeiten auch zeitlich festgelegte (familiäre) Care-Arbeiten mit zum Beispiel Schul- und Ferienzeiten beinhalten. Das komplexe Wissen, das zur Betreuung von Honigbienen notwendig ist, ist ein weiteres Hemmnis.

Durch die Mischtracht, die Honige aus der Stadt prägt, ist eine Vermarktung als lokales Produkt sinnvoll und üblich. Aufgrund der erzeugten Honigmengen erfolgt die Veredelung und Inverkehrbringung meist im (nebenerwerblichen) Direktvertrieb, also mit klimaschonender oder sogar klimaneutraler Lieferkette. Für die mit dem Bienenstand verbundenen Spezifika der Bienenhaltung bietet die Agrarinformatik technologische Lösungen, die sowohl der Sicherheit der Bienenstände als auch der Unterstützung der Imkernden dienen. optimierung den Gesundheitszustand von Tieren mittels Temperatur- und Gewichtsmessungen überwacht sowie emissionsbezogene Umweltschutzziele einbindet. Die für die klassische Bienenhaltung entwickelten Anwendungen und Systeme der Agrarinformatik können urbanen Neuimker*innen mit Handlungsempfehlungen imkerliche Entscheidungen erleichtern und dokumentieren helfen. Durch die Einbeziehung von Daten aus den Bienenstöcken wird minimalinvasives und damit bienenschonendes Imkern unterstützt, weil überflüssige Kontrollblicke vermieden werden. Der Rückgriff auf die digitale Infrastruktur erleichtert und ermöglicht Imkernden in der Stadt dabei den $\mathrm{Zu}-$ griff auf Anwendungen und Daten in Echtzeit.

Allerdings ist trotz aller Vorteile und innovativer Potenziale das smarte Imkern aus verschiedenen Gründen nicht unproblematisch. Werden die Daten über IoT-Netzwerke übermittelt, sind hiermit Fragen der Datensicherheit und des Datenschutzes verbunden, besonders wenn die Daten von Dritten verarbeitet oder 
genutzt werden können. Liefern Sensoren Daten in Echtzeit, lösen bereits Störungen im Datenstrom Diebstahl- oder Vandalismusalarm aus.

\section{Gemeinschaftliches Imkern}

Gemeinschaftliches Betreuen von Bienen auf der Basis einer solidarischen Produzenten-Konsumenten-Gemeinschaft kann Lösungen bieten, um interessierten Stadtbewohner*innen eine bessere Vereinbarkeit von Imkerei und urbanen Lebensstilen, z. B. hinsichtlich Beruf, Familie und weiteren Verpflichtungen zu er- kurzfristig angesetzt wurden. Der Kontakt mit Bienen stand im Vordergrund des Interesses und es führte zu Enttäuschungen, wenn durch anleitende Expert*innen ausschließlich theoretisches Wissen vermittelt wurde. Fluglochbeobachtungen als Sichtkontrolle von außen dagegen wurden nicht als Bienenkontakte gewertet. Diese imkerliche Monitoring-Methode war initialer Bestandteil der Treffen. Die Auswertung der von den Imkernden selbst gefilmten Interaktionen zwischen Menschen und Bienen zeigt, dass Imkernde die Kontakte mit Bienen an der offenen Bienenbeute überwiegend als ein besonderes Naturerlebnis erfuhren. Die jährlichen Honigschleudertage dagegen wurden auch ohne Bienenkontakt als zentrale Erlebnisse geschildert

\section{Solidarisch organisierte Produzenten-Konsumenten- Gemeinschaften bieten Lösungen für die Vereinbarkeit von Imkerei und urbanen Lebensstilen.}

möglichen. In der traditionell auf individuell Imkernde ausgerichteten Bienenhaltung stellt der Trend zum urbanen gemeinschaftlichen Imkern eine Sozialinnovation dar, die in Bremen von verschiedenen Institutionen angeboten wird. Die Variationen in Ausgestaltung und Praxen des gemeinschaftlichen Handelns unter Einsatz von agrarinformatischen Anwendungen zur Unterstützung in gemeinschaftlichen Imkerndengruppen werden im Folgenden an den Untersuchungsergebnissen aus unterschiedlichen Institutionen (Hochschulsport, Urban-GardeningProjekt, Bürgerzentrum) dargestellt.

\section{Motivationen zur Teilnahme}

Die Teilnehmenden der gemeinschaftlichen Imkergruppen nannten als Gründe für die Teilnahme den Wunsch, einen Beitrag gegen das Bienensterben und für den Umweltschutz zu leisten, grundsätzliches Interesse an nachhaltigem Arbeiten und ökologischen Aspekten, Achtsamkeit, aber auch ein generelles, familiär vorgeprägtes Interesse und das Ziel, selbst imkern zu können. Diese Motivationen decken sich mit denen des Deutschen Imkerbundes (DIB 2020a, S. 12) und sind ebenso wie jene zur Bienenhaltung in der Stadt überwiegend dem Spektrum von Naturschutz und Naturerlebnis zuzuordnen (Peters 2012; Fenske 2017, 2019; Heyer 2018; Lorenz und Stark 2015; Sulzner 2016; Moore und Kosut 2014). Die Erwartungen gemeinschaftlich Imkernder adressieren damit Ziele, die über den Naturschutz und die Lebensmittelerzeugung im Stadtraum auf eine weltanschauliche Transformation hin zu klimaneutralen Städten verweisen.

\section{Gemeinschaftliche Imkerpraxen}

Die Gruppen organisierten das Imkern über im Voraus festgelegte Treffen. Da imkerliche Eingriffe von Wetterfaktoren und Abläufen im Bienenstock abhängen, passten die lang- oder mittelfristig vereinbarten Termine nicht immer, so dass einige auch und erweiterten den Bienen-Fokus auf Honig und Trachtpflanzen. Die Teilnahme an imkerlichen Tätigkeiten rund ums Bienenjahr sensibilisierte die Teilnehmenden für regionalen Honig und damit klimaneutralen Honigkonsum.

Vor allem in Kurssystemen ist das gemeinschaftliche Imkern ein Mitimkern auf Anweisung der Anleiter*innen. Dies liegt zunächst an Zugangsbarrieren durch die Besitzverhältnisse und die formale Organisation. Stehen den beteiligten Imkernden nicht dieselben Zugangs- und Informationsressourcen zur Verfügung, entstehen Hierarchiegefälle, die sich im Fortgang der Kurse eher verstärkten als auflösten. Etablierte Verantwortungsgefüge formeller wie informeller Art prägen Zugangsstrukturen und -barrieren in institutionellen Gebäuden ebenso wie zu öffentlichen Flächen. Information spielt eine wesentliche Rolle gerade bezüglich infrastruktureller und praktischer Hintergrundinformationen, die Handlungsfähigkeit schaffen. Mit Blick auf die gleichberechtigt-gemeinschaftliche Bienenhaltung stellen etwa die veterinärrechtlichen Organisationsstrukturen, die eine Anmeldung der Bienenhaltung nur durch natürliche Personen vorsieht, eine bürokratische Hürde für diese soziotechnische Innovation dar.

\section{Potenziale und Hürden bei der Einbindung von IKT}

Den nichtamtlichen Hürden für gemeinschaftliche Verantwortungsübernahme kann durch die Einbindung von Informations- und Kommunikationstechnologien (IKT) in Form von agrarinformatischen Hilfssystemen ins gemeinschaftliche Imkern entgegengewirkt werden. Für den Austausch organisatorischer Informationen wurde in den Imkerndengruppen auf Initiative der Anleiter*innen ein etablierter Instant-Messaging-Dienst genutzt. Einzelne Sensoren wurden bereits von Anfang an in und an den Bienenbeuten angebracht. Diese wurden bis zum Ende der Feldforschung nur an einem Standort zur Datenaufzeichnung in Betrieb genommen, jedoch nicht zum gemeinschaftlichen Im- 
kern genutzt. Ein Grund kann - neben der fehlenden Routine die Motivation zum Besuch der Imkergruppen sein, die naturerlebnis- statt technikorientiert ist. Das Führen von Stockkarten, auf denen Beobachtungen und imkerliche Eingriffe für jeden Bienenstock datumsgenau dokumentiert werden, wurde erst mit der Einführung einer virtuellen Stockkarte an einem Standort einige Wochen vor Ende der Feldforschung eingeführt und erfolgte bis zum Ende der Kurse nur sporadisch. Stockkartenführung werden insgesamt von Imkernden, so zeigt auch die Analyse weiterer Feldforschungsdaten, als imkerferne Tätigkeit wahrgenommen, der nur Imkernde mit vielen Superorganismen nachgehen. Um alle Imkernden im gemeinschaftlichen Imkern auf denselben Informationsstand über die Bienen zu bringen und einer Stadt könnte über die Förderung der Stadtbienenhaltung und die Verwendung für eine intelligente Verwaltung der Imkerei hinausgehen. Eine solche Ausdehnung der urbanen Honigbienenhaltung und eine weitere Einbindung agrarinformatischer IoT-Anwendungen in die Systeme intelligenter Stadtentwicklungsprozesse würde allerdings das Risiko einer biodiversitätsfeindlichen Verengung des Blicks auf Tiere in menschlicher Haltung verschärfen.

Denn die Vorteile für die Honigbienenhaltung in urbanen Räumen - wenig Monokulturen und Pestizideinsatz - sind auch für Wildbienen wichtige (Über-)Lebensraumeigenschaften. So kommt die wachsende Honigbienendichte, die bisher lediglich zu einem Stichtag und nur auf den gesamten Stadtraum berech-

\section{Die Vernetzung der Honigbienenhaltung durch IoT-Technologien kann die Bedrohung der vulnerablen urbanen Wildbienenpopulationen weiter verschärfen.}

eine egalitäre Aufgabenteilung zu ermöglichen, ist sie im gemeinschaftlichen Imkern aber ratsam. Eine digitale Lösung optimiert hier die Terminplanung.

Während gemeinschaftliche Bienenhaltung als haushaltsübergreifende Wirtschaftsform in Imkervereinen erklärungsbedürftig ist, ist das Imkern mit Sensorbeuten dort leichter anschlussfähig, wird oft bereits diskutiert und teilweise schon erprobt. Für die neuen Imkernden steht das Naturerlebnis im Fokus, weshalb die Nutzung von IoT-Technologien, besonders von Technikkomponenten in Bienenbeuten, zunächst auf Ablehnung stößt. Hier können einfache Anwendungen und Plug-andPlay-Systeme Einstiegshürden abbauen. Zusätzlich müssten die Vorteile der Datensammlung über den Bienenstock und der Erstellung eines digitalen Zwillings für das gemeinschaftliche Imkern und die Funktion der Entscheidungsunterstützung auf Basis der Sensordaten betont werden.

\section{Fazit}

Der mehrdimensionale Beitrag der Honigbienenhaltung als Teil urbaner Agrikultur zur Klimaneutralität in Städten ist unbestritten. Der Einsatz von innovativen IKT-, IoT und KI-Anwendungen bietet dafür, gemeinsam mit der Sozialinnovation des gemeinschaftlichen Imkerns, organisatorische und entscheidungsunterstützende Möglichkeiten, z. B. Überwachungsfunktionen zum bienenschonenden Imkern. Insbesondere Vernetzung und Freigabe von imkereibezogenen Daten können gemeinschaftliches Imkern erleichtern bzw. es erst ermöglichen. Darüber hinaus können sie Bausteine für den Seuchenschutz sowie für die kontinuierliche und flächendeckende Dokumentation der Honigbienendichte sein. Die Datennutzung aus den Bienenstöcken net wird, aus Sicht des Wildbienenschutzes einer sich verschärfende Nahrungskonkurrenz für Wildbienen gleich. Die Flächen, die Wildbienen Nistmöglichkeiten und spezielle Futterpflanzenaussaaten durch Naturschutzakteure bieten, sind oft kommunale Mehrfachnutzungsflächen und stets der Mitnutzung durch Menschen, der Gefahr der Umnutzung oder des Abmähens zur Grünflächenpflege ausgesetzt.

Die Vernetzung der Honigbienenhaltung durch IoT-Technologien kann die Bedrohung der vulnerablen Wildbienenpopulationen weiter verschärfen, da ihr Vorkommen nur mit zeitaufwändigem und geschultem Monitoring auf entsprechenden Flächen beobachtet werden kann. Werden die Daten des Wildbienen-Monitorings aber in Verbindung mit der Bestäubungsleistung der Honigbienen gebracht (die über Analysen der Nahrungspflanzenanteile im Honig erhoben werden) oder mit der tatsächlichen Honigbienenstandortdichte verknüpft (die für Imkernde auch aufgrund des zu erwartenden Honigertrags relevant ist), kann die Verwendung von IoT-Anwendungen und -Daten auch den Natur- und Artenschutz unterstützen und so einen Beitrag zu intelligenten und klimaneutralen Städten mit großer Biodiversität in Fauna und Flora leisten.

\section{Erklärung zur Forschungsförderung \\ Die Forschungen für diesen Beitrag wurden gefördert durch das BMBF im Rahmen des Projekts „Urban Agriconnect: Dynamisches Modell des gesellschaft- lichen Wandels zur Bioökonomie im urbanen Raum“.}

\section{Literatur}

Bornemann, Basil; Weiland, Sabine (2019): Empowering people-democratising the food system? Exploring the democratic potential of food-related empowerment forms. In: Politics and Governance 7 (4), S. 105-118. https://doi.org/10.17645/pag.v7i4.2190 
DIB - Deutscher Imkerbund e. V. (2020 a): Jahresbericht 2019. Online verfügbar unter https://deutscherimkerbund.de/userfiles/DIB_Pressedienst/ Jahresbericht_2019.pdf, zuletzt geprüft am 23.11.2020.

DIB (2020 b): DIB Aus- und Fortbildung. Frauen in der Imkerei. Online verfügbar unter https://deutscherimkerbund.de/176-DIB_Nachwuchsfoerderung_ Frauen_in_der_Imkerei, zuletzt geprüft am 07.12.2020.

Dineva, Kristina; Atanasova Tatjana (2017): Computer system using internet of things for monitoring of bee hives. In: Proceedings of $17^{\text {th }}$ international multidisciplinary scientific GeoConference SGEM. Sofia, Bulgaria, 27.-29.11.2017, pp. 169-176. https://doi.org/10.5593/sgem2017H/63/S25.022

Dzierzon, Johann (1855): Neue verbesserte Bienenzucht des Pfarrers Dzierzon. Brieg: C. Schwartz.

Epstein, Katharina (2019): Einleitung. In: Katharina Epstein (Hg.): De partibus animalium. Aristoteles. Darmstadt: Wissenschaftliche Buchgesellschaft.

Fenske, Michaela (2017): Narrating the swarm. Changing metanarratives in times of crisis. In: Narrative Culture 4 (2), S. 130-152. https://doi.org/10.13110/ narrcult.4.2.0130

Fenske, Michaela (2019): Retten und gerettet werden. Europäische Honigbienen und Menschen im urbanen Resonanzraum. In: Siegfried Becker und Sonja Windmüller (Hg:): Hessische Blätter für Volks- und Kulturforschung 52, S. 93-107.

Heyer, Marlis (2018): Von Menschenkindern und Honigbienen. Multispecies-Perspektiven auf Begegnungen am Bienenstand. Würzburg: Universität Würzburg. https://doi.org/10.25972/OPUS-16707

Lecocq, Antoine; Kryger, Per; Vejsnæs, Flemming; Bruun Jensen, Annette (2015): Weight watching and the effect of landscape on honeybee colony productivity. Investigating the value of colony weight monitoring for the beekeeping industry. In: Plos One 10 (7), S. e0132473. https://doi.org/10.1371/journal. pone. 0132473

Lorenz, Stephan; Stark, Kerstin (2015): Die summende Stadt. Zum Stadtimkereitrend in Berlin. In: Stephan Lorenz und Kerstin Stark (Hg.): Menschen und Bienen. Ein nachhaltiges Miteinander in Gefahr. München: Oekom, S. 217-228.

Moore, Lisa; Kosut, Mary (2014): Among the colony. Ethnographic fieldwork, urban bees and intra-species mindfulness. In: Ethnography 15 (4), S. 516-539. https://doi.org/10.1177/1466138113505022

Müller, Christa (Hg.) (2011): Urban Gardening. Über die Rückkehr der Gärten in die Stadt. München: Oekom.

Peters, Kathryn (2012): Keeping bees in the city. Disappearing bees and the explosion of urban agriculture inspire urbanites to keep honeybees. Why city leaders should care and what they should do about it. In: Drake Journal of Agricultural Law 17 (3), S. 597-644.

Sulzner, Raffaela (2016): Von den guten Bienen. Mensch-Tier-Begegnungen in der urbanen Imkerei Wiens. In: Lukasz Nieradzik und Brigitta Schmidt-Lauber (Hg): Tiere nutzen. Ökonomien tierischer Produktion in der Moderne. Innsbruck: StudienVerlag, S. 183-194.

von der Ohe, Werner; Lüken, Dorothee; Suckrau, Iris; Bartz, Torsten; Gunter, Jan (2017): Bienenvölker im Umweltmonitoring. Stadt/Land-Vergleich zu Rückständen in Bienenprodukten. Leipziger Blaue Hefte 9 (3), S. 345-347.

Zabasta, Anatolijs; Kunicina, Nadezda; Kondratjevs, Kaspars; Ribickis, Leonids (2019): I0T approach application for development of autonomous beekeeping system. In: Proceedings of International Conference in Engineering Applications (ICEA), Sao Miguel, Portugal, 08.-11. 07.2019, S.1-6. https://doi. org/10.1109/CEAP.2019.8883460
Zacepins, Aleksejs; Kviesis, Armands; Ahrendt, Peter; Richter, Uwe; Tekin, Saban; Durgun, Mahmut (2016): Beekeeping in the future. Smart apiary management. In: Proceedings of 17th International Carpathian Control Conference (ICCC), Tatranská Lomnica, Slowakei, 29. 05.2016, S. 808-812. https://doi.org/10.1109/ Carpathiancc.2016.7501207

Zezza, Alberto; Tasciotti, Luca (2010): Urban agriculture, poverty, and food security. Empirical evidence from a sample of developing countries. In: Food Policy 35 (4), S. 265-273. https://doi.org/10.1016/j.foodpol.2010.04.007

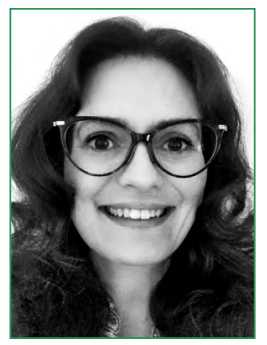

\section{MARIE-HELENE WICHMANN}

hat von 2016-2020 im BMBF-geförderten Projekt „Urban Agriconnect. Dynamisches Modell des gesellschaftlichen Wandels zur Bioökonomie im urbanen Raum" gearbeitet. Sie ist wissenschaftliche Mitarbeiterin am Institut für Ethnologie und Kulturwissenschaft (IfEK) und forscht u. a. im Bereich der Human Animal Studies. 